\title{
Strain relaxation of CdTe films growing on lattice-mismatched substrates
}

\author{
Zhixun Ma • Kin Man Yu • Wladek Walukiewicz • \\ Peter Y. Yu • Samuel S. Mao
}

Received: 25 October 2008 / Accepted: 3 March 2009 / Published online: 11 April 2009

(C) The Author(s) 2009. This article is published with open access at Springerlink.com

\begin{abstract}
We have deposited CdTe films by laser-assisted epitaxy approach and investigated the influence of substrate and film thickness on the film properties. Grown on $\mathrm{Si}(001), \mathrm{GaAs}(001)$, and quartz substrates; the CdTe films exhibit preferential orientation along the cubic $\mathrm{CdTe}(111)$ direction. When the films are thin $(<500 \mathrm{~nm})$, a blueshift of the band gap and splitting of valence bands were observed. These results are attributed to the existence of residual strains induced by mismatch of the film lattice constant with that of the substrate, and by their difference in thermal expansion coefficients. The bulk band-gap energy of $1.5 \mathrm{eV}$ was achieved on the surface of thick CdTe films grown on $\mathrm{Si}(001)$ substrate, indicating that strain was almost completely relaxed in this case. Our results demonstrate that by a proper selection of substrate and film thickness it is possible to grow film semiconductors with band gap approaching those of bulk crystals.
\end{abstract}

PACS 61.05.cp $\cdot 68.37 . \mathrm{Hk} \cdot 68.55 . \mathrm{ag} \cdot 73.20 . \mathrm{At}$

\section{Introduction}

Much effort has been devoted to the development of roomtemperature radiation detectors for defense applications [1-3]. Compound semiconductors based on materials, such

Z. Ma · K.M. Yu · W. Walukiewicz · P.Y. Yu · S.S. Mao ( $ه)$ Lawrence Berkeley National Laboratory, Berkeley, CA 94720 , USA

e-mail: ssmao@lbl.gov

P.Y. Yu

Department of Physics, University of California, Berkeley, CA 94720, USA as cadmium telluride (CdTe), hold great promise for these applications. In order for a semiconductor to operate as radiation detectors at room temperature, they need to possess a number of features: (1) a large enough band gap and low enough carrier density to minimize current noises; (2) free from traps that act to reduce the useful signal; (3) higher carrier mobilities to produce pulses with fast rise time; (4) a high atomic number element for good stopping power of high energy radiation; and (5) a long carrier lifetime to allow efficient charge collection. So far high-quality bulk single crystals of CdTe-based semiconductors appear to have many of these properties. A wide variety of techniques [4] have been used to grow these bulk crystals, such as the Bridgman and Czochralski methods.

On the other hand, film semiconductors have the advantage that they can be grown much faster than bulk crystals. In particular, CdTe films can be grown by a number of techniques, including molecular beam epitaxial (MBE) [5, 6], metalorganic chemical vapor deposition (MOCVD) [7], and laser-assisted epitaxy or pulsed-laser deposition (PLD) [810]. In general, thin films grown epitaxially on a latticematched substrate have been found to have crystalline quality comparable or even better than of the bulk crystals. For example, AlGaAs thin films grown on lattice-matched GaAs substrates have higher quality than the corresponding bulk crystals. CdTe films have so far been grown primarily on lattice-mismatched substrates. These films are usually strained because of the difference in lattice constants and/or coefficients of thermal expansion between the epilayer and the substrate. As a result, these thin films often have poor electronic properties as compared to the corresponding bulk material. It is well known that extended defects, such as dislocations, can form in epilayers grown on lattice-mismatched substrates. However, it is possible to localize these defects at the interfacial region [11]. Thus, by 
increasing the film thickness, it may be possible to grow strain-relaxed epitaxial films with crystal quality approaching that of the bulk crystal [12]. We have used the laserassisted epitaxy technique, which is a high-vacuum film deposition method, to grow CdTe films on a number of latticemismatched substrates. We demonstrate that micron-thick single crystalline CdTe films can be grown on $\mathrm{Si}(001)$ substrates with the band gap approaching that of the bulk single crystal.

\section{Experimental details}

The CdTe films were grown on $\mathrm{Si}(001), \mathrm{GaAs}(001)$, and quartz substrates at various substrate temperatures $\left(T_{\mathrm{S}}\right)$ in a vacuum of $\sim 3 \times 10^{-7}$ torr using the laser-assisted epitaxy technique. The excitation source is a $\mathrm{KrF}$ excimer laser emitting $30 \mathrm{~ns}$ pulses and operating at a wavelength of $248 \mathrm{~nm}$. The pulse energy of the laser beam is approximately $160 \mathrm{~mJ}$ and the repetition rate is $5 \mathrm{~Hz}$. CdTe target with purity of 99.999\% was mounted on a rotating target holder and ablated to produce the precursor vapor. The substrates were attached to a substrate holder and heated at $500^{\circ} \mathrm{C}$ for more than $10 \mathrm{~min}$ to thermally remove any contamination before cooling to the desired temperature for film deposition. The distance between the target and the substrates was about $5 \mathrm{~cm}$.

The CdTe films were characterized by modulated photoreflectance (PR) spectroscopy, X-ray diffraction (XRD), and scanning electron microscopy (SEM). In the PR measurements, the samples were excited with a $325 \mathrm{~nm}$ pump beam chopped at $310 \mathrm{~Hz}$. The probe beam came from a highpressure tungsten lamp after being dispersed by a monochromator with a $600 / \mathrm{mm}$ grating. The reflected light was detected by a Si photodiode and a lock-in amplifier. XRD measurements were carried out using the $\mathrm{Cu} \mathrm{K}_{\alpha}$ radiation with a Siemens D5000 diffractometer.

\section{Experimental results and discussion}

Since the lattice constant of CdTe (6.480 $\AA$ ) is much larger than that of GaAs $(5.653 \AA)$ and $\mathrm{Si}(5.431 \AA),(111)$ oriented cubic CdTe films have been found to grow on these (001) oriented substrates [5-10] in order to minimize the surface free energy. We discuss the properties of CdTe films grown on these two substrates separately.

\subsection{CdTe films of different thickness grown on $\mathrm{Si}(100)$}

Figure 1a shows the typical XRD patterns of CdTe films grown on $\operatorname{Si}(001)$ substrate at $T_{\mathrm{s}}=300^{\circ} \mathrm{C}$ and with film thickness less than $1 \mu \mathrm{m}$. The dominant domain orientation
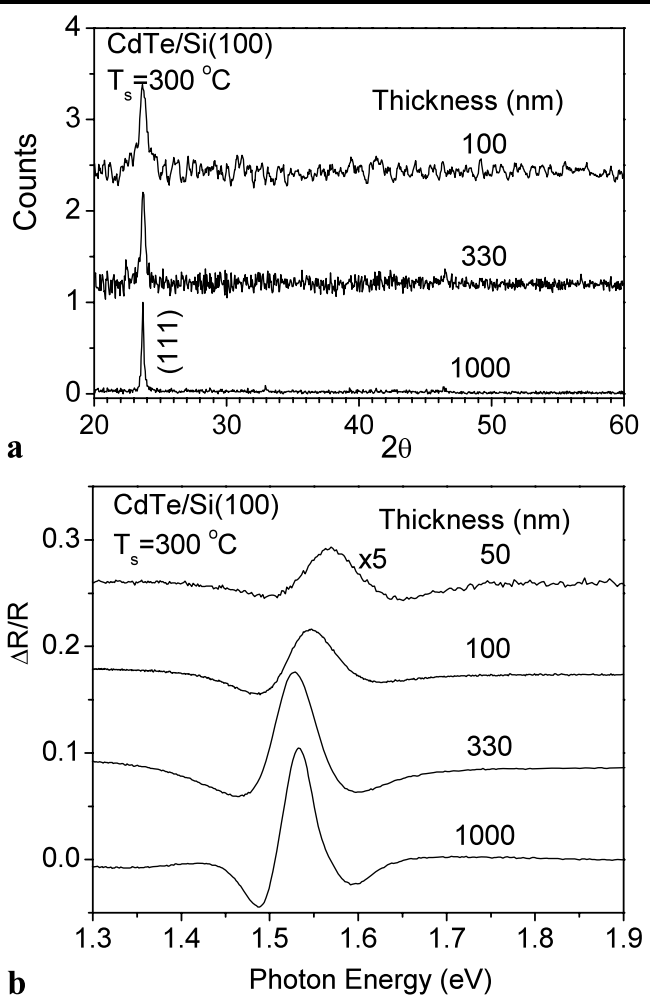

Fig. 1 (a) XRD patterns and (b) PR spectra of CdTe films with different thicknesses

of the CdTe film is found to be [111]. The width of the (111) diffraction peak is found to be broader for the thinner films indicating that there are either more defects or the crystallites are smaller in the thinner films. With increasing film thickness the lattice constant of CdTe as determined from the XRD become closer to that of the perfect bulk crystal showing that strain relaxation occurs as thickness increases.

The XRD results are consistent with the results derived from the PR spectra shown in Fig. 1b. The existence of strain in the CdTe film is manifested in the PR spectra by shifts in the band gap as the film thickness is increased. The band-gap energies can be obtained by fitting the measured PR curves using sums of Lorentzians [13], $\Delta R / R=$ $\operatorname{Re}\left[A e^{i \Phi}\left(E-E_{0}+i \Gamma\right)^{-m}\right]$, where $A$ is the amplitude, $\Phi$ is the phase angle, $E_{0}$ is the onset energy of interband absorption (to be referred to as the "band-gap energy"), $\Gamma$ is the broadening factor, and $m=2.5$ for bulk semiconductors. We found that the curves in Fig. 1b could be fitted well by a sum of two Lorentzians, as illustrated in Fig. 2. This suggests that the band gap is split by strain into two transitions. The inset of Fig. 2 shows that, when the film thickness increases from 50 to $1000 \mathrm{~nm}$, the split decreases in energy from 49 to $18 \mathrm{meV}$ while the fundamental band gap decreases from 1.574 to $1.513 \mathrm{eV}$. We note that the last value approaches the bulk value of $1.50 \mathrm{eV}[14,15]$. The decrease in both the splitting and the band-gap energy indicates that the strain is relaxed to some extent with increasing the film thickness. 


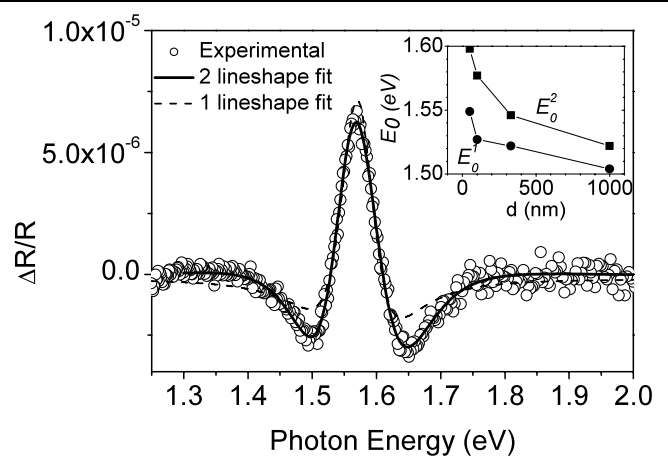

Fig. 2 PR spectrum of the $C d T e / S i(100)$ with thickness of $50 \mathrm{~nm}$. The inset is the fitting results of the band-gap split as function of film thickness

CdTe films grown on $\mathrm{Si}(001)$ typically have been shown to have two orthogonal crystalline domains [6]: $\mathrm{CdTe}[1-$ 10]\| $\mathrm{Si}[110]$ and $\mathrm{CdTe}[11-2] \| \mathrm{Si}[1-10]$, in which the lattice mismatch is reduced to 16 and $3 \%$ along the [1-10] and [11-2] directions, respectively. The domains are compressed in both directions $[6,16]$, which is different from the case of CdTe grown on $\operatorname{GaAs}(001)$ [17]. In addition to the strain introduced by the lattice-mismatch, a difference in the thermal-expansion coefficients of the epitaxial layer and the substrate can introduce thermal strains. For the $\mathrm{CdTe} / \mathrm{Si}$ system, the CdTe layer can be biaxially strained because the thermal expansion coefficient of CdTe $\left(4.8 \times 10^{-6} / \mathrm{K}\right)$ is greater than that of $\mathrm{Si}\left(2.6 \times 10^{-6} / \mathrm{K}\right)$. When the sample is cooled from the growth temperature to room temperature, the CdTe film undergoes a tensile strain. These two types of strains are primarily responsible for changing the band gap energies of the CdTe films from that of the bulk crystals.

In general, a strain can be decomposed to a hydrostatic and a shear component. The former leads to a change of the fundamental band-gap energy while the latter causes a splitting of the heavy- and light-hole bands. Even though the [111] orientation of the $\mathrm{CdTe}(111)$ domains is, in principle, parallel to the [001] orientation of the $\mathrm{Si}(001)$ substrate, the shear strain can still change from site to site due to the presence of several equivalent [110] axes within the $\mathrm{Si}(001)$ plane. Since XRD results show that there is practically no change in the lattice parameter of the CdTe films along [111] direction as a function of film thickness, the CdTe bandgap split cannot be attributed to a uniaxial strain along the [111] direction [18]. However, an increase in the fundamental band-gap energy in the thin CdTe film with respect to that of a bulk CdTe suggests that a compressive biaxial strain is present in the CdTe epilayers [19].

\subsection{CdTe films grown on $\mathrm{Si}(100)$ at different substrate temperatures}

A series of thick CdTe samples ( $>3 \mu \mathrm{m}$ thick) were grown at different temperatures $T_{\mathrm{S}}$ on $\mathrm{Si}(001)$ substrates in order to study the evolutions of the crystal quality as well as the band-gap energy as a function of $T_{\mathrm{s}}$. We found that the CdTe films grown at below $100^{\circ} \mathrm{C}$ are amorphous with band gap of about $1.6 \mathrm{eV}$ at room temperature. As $T_{\mathrm{S}}$ is increased to $100^{\circ} \mathrm{C}$ and above, CdTe crystallites oriented preferentially along the [111] direction are formed as shown by the XRD results in Fig. 3a. It is clear that the CdTe (111) diffraction peak is located at lower angles when grown at 100 and $150^{\circ} \mathrm{C}$ as compared to the samples grown at higher $T_{\mathrm{s}}$. This implies that the lattice constant perpendicular to the growth plane is increased at lower $T_{\mathrm{s}}$. In the meantime the band-gap energy is increased at low $T_{\mathrm{S}}$ as shown by the PR spectra in Fig. 3b. Multiple transitions are observed for the samples grown at 250 and $300^{\circ} \mathrm{C}$, indicating the residual strain becomes larger at higher growth temperatures. On the other hand, the transition energies may be related to the quantum confinement effect of the smallcrystallites. Figure 3(c) shows SEM images of CdTe films grown under different temperatures. The average diameter of CdTe crystallites increases from about $35 \mathrm{~nm}$ to $170 \mathrm{~nm}$ as Ts increases from 100 to $300^{\circ} \mathrm{C}$. For a crystallite with $35 \mathrm{~nm}$ in diameter embedded in amorphous CdTe with $1.6 \mathrm{eV}$ band-gap energy, the confinement energy is about $3 \mathrm{meV}$, much smaller than the observed increase at $T_{\mathrm{s}}=100^{\circ} \mathrm{C}$. However, it is possible that smaller crystallites exist in films grown at lower temperatures, the resulting quantum confinement effect can therefore contribute to the observed high energy shift of the band-gap energy at 100 and $150^{\circ} \mathrm{C}$. At higher growth temperatures $\left(>200^{\circ} \mathrm{C}\right)$, quantum confinement effects would be negligible.

The transition energies can be obtained by fitting the PR spectra with Lorentzians. The PR spectra can be fitted with two Lorentzians for the samples grown at 100 and $150^{\circ} \mathrm{C}$, and three Lorentzians for the samples grown at $T_{\mathrm{s}} \geq 200^{\circ} \mathrm{C}$. The results obtained by fitting the experimental PR spectra are summarized in Fig. 4a. As $T_{\mathrm{S}}$ increases, the lowest transition energy $\left(E_{0}\right)$ continuously decreases and reaches a constant value whereas the higher ones ( $E_{01}$ and $\left.E_{02}\right)$ continue to increase with $T_{\mathrm{s}}$.

To understand the results in the thick CdTe films, we propose that these films actually consist of two layers: one is a thin layer (of thickness comparable to the critical layer thickness) adjacent to the interface which is highly strained but not dislocated, and the other is a thick top layer where the strain has mostly been relaxed by dislocations. The $E_{0}$ transition comes from the strain-relaxed top layer while the higher energy transitions $\left(E_{01}\right.$ and $\left.E_{02}\right)$ arise from the strained layer at the interface. At $T_{\mathrm{S}}<100^{\circ} \mathrm{C}$ only amorphous CdTe forms and the band gap of the amorphous phase is usually larger than that of the crystalline phase. When $T_{\mathrm{s}}>100^{\circ} \mathrm{C}$ crystallites start to form but their strain cannot be relaxed effectively by the low substrate temperature. With 


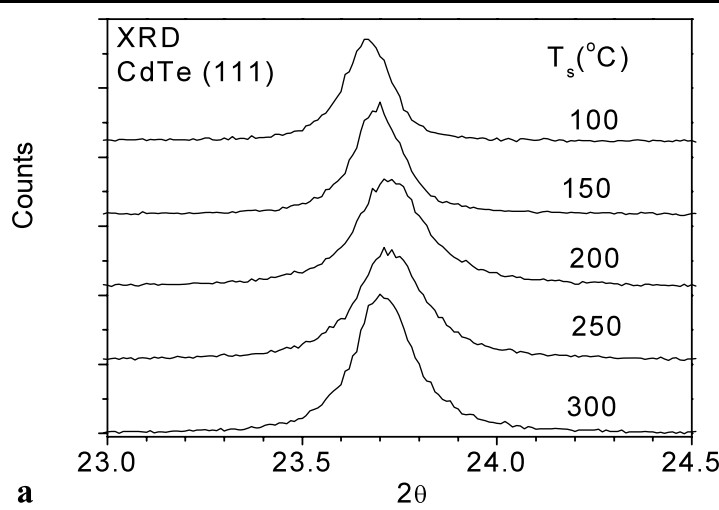

a
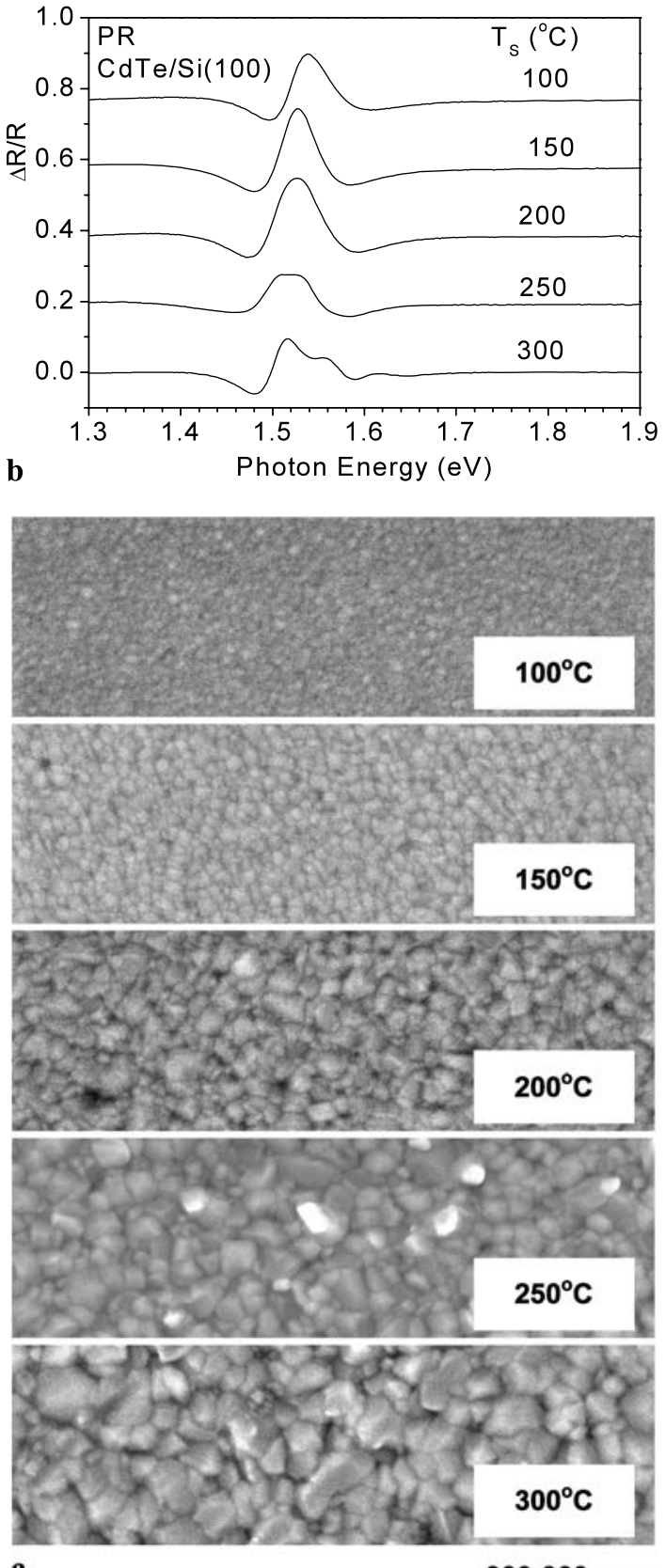

c

$300,300, \mathrm{~nm}$

Fig. 3 (a) (111) XRD peaks, (b) PR spectra, and (c) SEM images of the thick $\mathrm{CdTe} / \mathrm{Si}(100)$ films grown at different temperatures

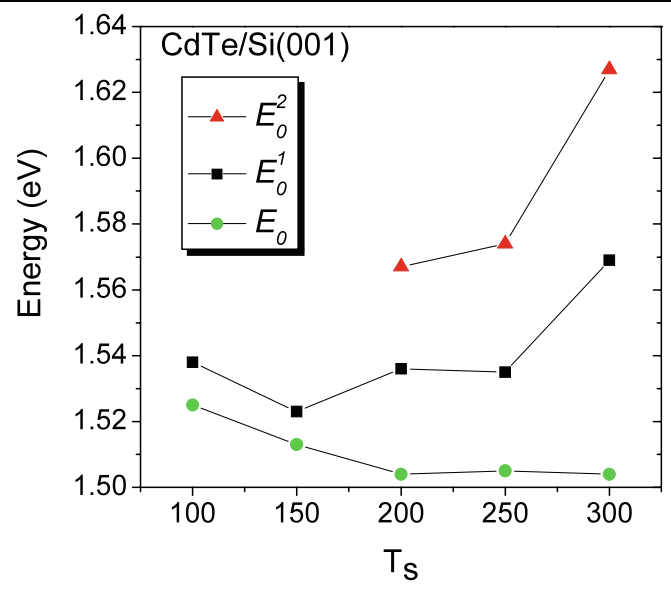

Fig. 4 The transition energies obtained by fitting the curves in Fig. 3b using Lorentzian lineshape

increasing $T_{\mathrm{S}}\left(>200^{\circ} \mathrm{C}\right)$, the strained thin layer near the interface becomes more strained due to increase in the thermal stress resulting in an increased split between the heavyand light- hole states as shown by $E_{01}$ and $E_{02}$ in Fig. 4a. Meanwhile, the dislocation starts to relax the strain of the crystallites far from the interface. As a result, the $E_{0}$ transition decreases in energy and reaches a constant value. This explanation is in agreement with the XRD results in Fig. 3a that shows an increase in the out-of-plane lattice constant at $T_{\mathrm{S}}<200^{\circ} \mathrm{C}$ due to the residual strains existing in the films grown at low $T_{\mathrm{s}}$.

We have considered the possibility that the increase of $E_{0}$ at low $T_{\mathrm{s}}\left(<200^{\circ} \mathrm{C}\right)$ may partly be due to quantum confinement effects [20] because the size of the crystallites is usually smaller at low $T_{\mathrm{s}}$. The quantum confinement effect of these smaller crystallites can contribute to the high-energy shift of the band gap as observed for the samples grown at 100 and $150^{\circ} \mathrm{C}$. However, at higher growth temperatures $\left(>200^{\circ} \mathrm{C}\right)$, quantum confinement effects on band gap should be negligible because of the large crystallite sizes. Strain relaxation in the top layer of the films grown at higher temperature is inferred by comparing the inset of Fig. 2 with Fig. 4 . For the films with thickness less than $1 \mu \mathrm{m}$ as in Fig. 2, the strain still exists throughout the film, thus only two transitions ( $E_{0}^{1}$ and $E_{0}^{2}$ ) have been observed. With increasing film thickness $(>3 \mu \mathrm{m})$ the strain in the top layer has been relaxed resulting in the observation of three transitions $\left(E_{0}\right.$, $E_{0}^{1}$ and $E_{0}^{2}$ ), where $E_{0}$ transition comes from the strain-free top layer. In the inset of Fig. 2 this strain relaxation continues even as the film thickness is increased leading to continued decrease in the value of $E_{0}$. In the case of Fig. 4, the film is already quite thick so the strain in the top layer is already partly relaxed. As the growth temperature is increased this strain is further relaxed. However, the strain relaxation saturates at $T_{\mathrm{S}}$ above $200^{\circ} \mathrm{C}$ as suggested by the value of $E_{0}$.

The above results suggest that thick films grown at temperatures above $200^{\circ} \mathrm{C}$ will achieve the maximum amount 

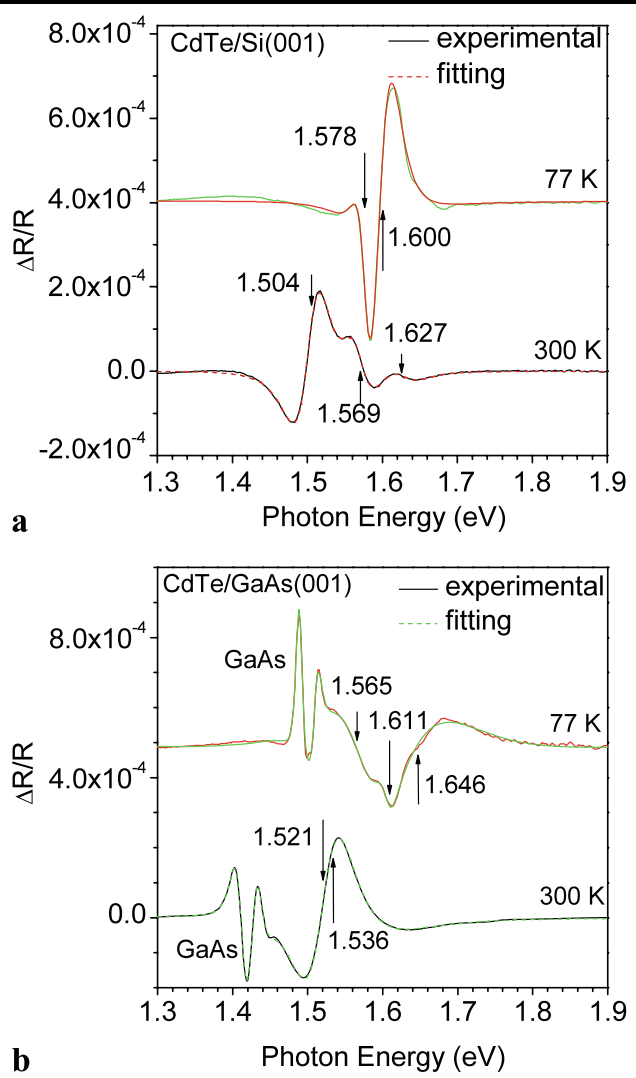

Fig. 5 PR spectra of (a) CdTe/Si(100) and (b) CdTe/GaAs(100) measured at $77 \mathrm{~K}$ and room temperature. The transition energies are obtained by fitting with the Lorentzian lineshape

of strain relaxation in their top layers. This conclusion is verified in Fig. 5a which shows the PR spectra of a $3 \mu \mathrm{m}$ thick CdTe film grown at $300^{\circ} \mathrm{C}$ on $\mathrm{Si}(001)$, measured at two different temperatures. The strong feature at 1.504 and $1.600 \mathrm{eV}$ at 300 and $77 \mathrm{~K}$, respectively, are nearly equal to the band-gap energies of bulk CdTe at the corresponding temperatures $(1.50$ and $1.60 \mathrm{eV}$ at 300 and $77 \mathrm{~K}$, respectively). These results indicate that this CdTe film is indeed nearly strain free. The high energy features at 1.569 and $1.627 \mathrm{eV}$ at $300 \mathrm{~K}$ are attributed to the splitting of heavy- and light-hole states due to the large shear strain in the thin layer at the interface as discussed earlier. At $77 \mathrm{~K}$, however, the PR spectrum can be well fitted by only two Lorentzians, indicating that the shear strain is actually smaller at low temperature. More importantly, the fundamental band-gap energy of the strained interface layer shifts in opposite direction relative to the bulk band gap at 300 and $77 \mathrm{~K}$. This difference is a manifestation of the opposite signs of the thermal-strain- and lattice-mismatch-induced strain. At $300 \mathrm{~K}$, the CdTe lattice is compressed as a result of the larger lattice constant of $\mathrm{CdTe}$ than that of silicon, resulting in a blueshift. At $77 \mathrm{~K}$, the CdTe lattice becomes tensilely strained since the thermal expansion coefficient of CdTe is

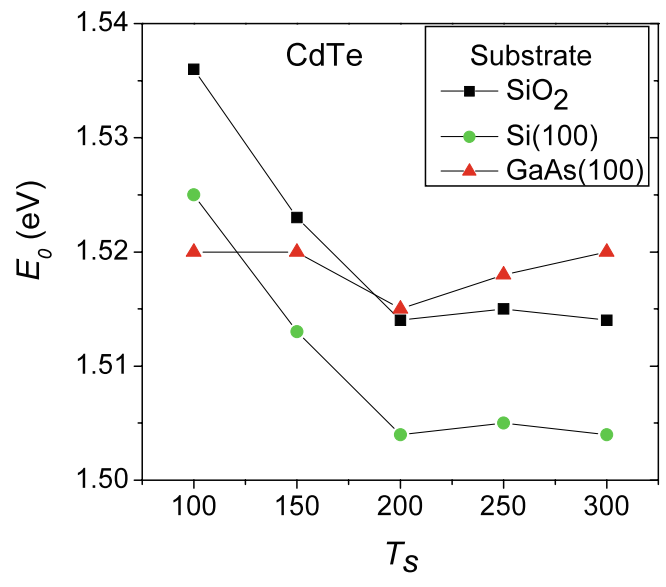

Fig. 6 Comparison of $E_{0}$ transition of thick CdTe films grown on $\mathrm{Si}(100), \mathrm{GaAs}(100)$, and quartz substrates

1.8 times larger than that of silicon, producing a redshift of the band-gap energy.

\subsection{CdTe films grown on $\operatorname{GaAs}(100)$ substrates}

Unlike CdTe films grown on $\mathrm{Si}(100)$, the CdTe domains in the $\mathrm{CdTe}(111) \| \mathrm{GaAs}(001)$ films are compressed along the [1-10] direction while extended along the [110] direction [12]. Furthermore, CdTe's thermal expansion coefficient $\left(4.8 \times 10^{-6} / \mathrm{K}\right)$ is smaller than GaAs $\left(6.0 \times 10^{-6} / \mathrm{K}\right)$. Thus, the lattice-mismatch-induced strain and the thermal strain of CdTe film grown on GaAs have opposite effects on its band-gap energy. Competition between these two effects make it difficult to predict qualitatively the behavior of the band-gap energy and split in CdTe films grown on GaAs(001) substrates.

Figure $5 b$ shows the PR spectra of a CdTe/GaAs(001) sample grown at $300^{\circ} \mathrm{C}$. The dominant structures at $1.521 \mathrm{eV}$ $(300 \mathrm{~K})$ and at $1.611 \mathrm{eV}(77 \mathrm{~K})$ are larger than those of the $\mathrm{CdTe} / \mathrm{Si}(001)$ sample shown in Fig. 5a. The multiple transitions induced by strains are also more prominent at $77 \mathrm{~K}$. The split states are located on the either side of the main feature at 1.566 and $1.646 \mathrm{eV}(77 \mathrm{~K})$. This indicates that the shear strain in the CdTe layer is larger at low temperature, which is different from that of the $\mathrm{CdTe} / \mathrm{Si}(001)$ sample. The larger fundamental band gap of $\mathrm{CdTe} / \mathrm{GaAs}(001)$ sample can be explained by the larger thermal expansion coefficient of CdTe than that of GaAs substrate leading to a compressive strain.

\subsection{Comparison between properties of CdTe films grown} on different substrates

We have also grown CdTe thin films on quartz substrates. Details of the results on these films will be presented elsewhere. We plotted in Fig. 6 the transition energy $E_{0}$ of CdTe 
films grown on different substrates as a function of $T$ so we can compare the substrate effect on the band-gap energy. It is interesting to note that, at low $T_{\mathrm{s}}, E_{0}$ is almost independent of $T_{\mathrm{s}}$ for the CdTe/GaAs(001) sample while $E_{0}$ decreases with increasing $T_{\mathrm{s}}$ for the samples grown both on $\mathrm{Si}$ and quartz. At higher $T_{\mathrm{s}}$, the larger $E_{0}$ values in the $\mathrm{CdTe} / \mathrm{GaAs}(001)$ and $\mathrm{CdTe} / q u a r t z$ films suggest that they both have larger strains than the $\mathrm{CdTe} / \mathrm{Si}(001)$ film. The closeness of the $E_{0}$ values in the latter films to those of the bulk crystals indicates that strain is completely relaxed in them. It should be noted that this finding applies only to the top layer of thick CdTe films grown on $\mathrm{Si}(001)$ substrate since the thin bottom layer near the substrate remains highly strained.

\section{Conclusions}

Using laser-assisted epitaxy approach, CdTe films of varying thickness have been fabricated on $\mathrm{Si}(001), \mathrm{GaAs}(001)$, and quartz substrates at different growth temperatures. All films, except for the almost amorphous ones grown at temperature below $100^{\circ} \mathrm{C}$, show a preferential orientation with (111) axis of the CdTe zincblende along the growth direction. A blueshift of the fundamental band gap with respect to the bulk value is observed in photoreflectance spectra of the thinner films $(<500 \mathrm{~nm})$ as well as the films grown at low growth temperatures $\left(<200^{\circ} \mathrm{C}\right)$ and attributed to residual compressive strain and/or quantum confinement effect of the small crystallites. Multiple transitions have been attributed to strained-induced splitting of heavy-hole and light-hole states of CdTe layer close to substrate. With increasing film thickness and growth temperature the strains in the top layers are largely relaxed; as a result, the band-gap is reduced. An important finding of the present work is that the strain in thick CdTe films grown on $\mathrm{Si}(001)$ substrate at temperatures above $200^{\circ} \mathrm{C}$ can be well relaxed so that their band gap approaches the value of bulk CdTe crystals. This makes it possible to substitute bulk crystals with crystalline CdTe films for the purpose of studying their applications, such as radiation detectors.
Acknowledgements This research has been supported by the U.S. Department of Energy, NNSA/NA-22, under Contract No. DE-AC02$05 \mathrm{CH} 11231$.

Open Access This article is distributed under the terms of the Creative Commons Attribution Noncommercial License which permits any noncommercial use, distribution, and reproduction in any medium, provided the original author(s) and source are credited.

\section{References}

1. A. Owens, J. Sychrotron Rad. 13, 143 (2006)

2. A. Owens, A. Peacock, Nucl. Instrum. Methods Phys. Res. A 531, 18 (2004)

3. D.S. McGregor, H. Hermon, Nucl. Instrum. Methods Phys. Res. A 395, 101 (1997)

4. P. Capper, A.W. Brinkman, Properties of Narrow Gap CadmiumBased Compounds, ed. by P. Capper, London, p. 369

5. C. Fontaine, J.P. Galliard, S. Magli, A. Million, J. Piaguet, Appl. Phys. Lett. 50, 903 (1989)

6. Y. Xin, N.D. Browning, S. Rujirawat, S. Sivananthan, Y.P. Chen, P.D. Nellist, S.J. Pennycook, J. Appl. Phys. 84, 4292 (1998)

7. H. Nishino, Y. Nishijima, J. Cryst. Growth 167, 488 (1996)

8. S. Neretina, R.A. Hughes, N.V. Sochinski, M. Weber, K.G. Lynn, J. Wojcik, G.N. Pearson, J.S. Preston, P. Mascher, J. Vac. Sci. Technol. A 24, 606 (2006)

9. J.J. Dubowski, D.F. Williams, P.B. Sewell, P. Norman, Appl. Phys. Lett. 46, 1081 (1985)

10. W.P. Shen, H.S. Kwok, Appl. Phys. Lett. 65, 2162 (1994)

11. V.H. Etgens, M. Sauvage-Simkin, R. Pinchau, J. Massies, N. Jedrecy, A. Waldhauer, S. Tatarenko, P.H. Jouneau, Phys. Rev. B 47, 10607 (1993)

12. H. Tatsuoka, H. Kuwabara, Y. Nakanishi, H. Fujiyasu, J. Appl. Phys. 68, 4592 (1990)

13. F.H. Pollak, H. Shen, Mater. Sci. Eng. R10, 275 (1993)

14. J.R. Mei, V. Lemos, Solid State Commun. 52, 785 (1984)

15. M.S. Han, T.W. Kang, T.W. Kim, Solid State Commun. 105, 709 (1998)

16. H. Tatsuoka, H. Kuwabara, Y. Nakanishi, H. Fujiyasu, J. Cryst. Growth 129, 686 (1992)

17. H.A. Mar, K.T. Chee, N. Salansky, Appl. Phys. Lett. 44, 237 (1984)

18. S. Jiménez-Sandoval, M. Meléndez-Lira, I. Hernández-Calderón, J. Appl. Phys. 72, 4197 (1992)

19. C.G. van de Walle, Phys. Rev. B 39, 1871 (1989)

20. S. Jiménez-Sandoval, M. Meléndez-Lira, I. Hernández-Calderón, J. Appl. Phys. 72, 4179 (1992) 